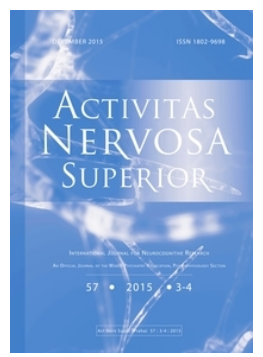

ANS: Journal for Neurocognitive Research Homepage:

www.activitas.org

IDEAS \& PERSPECTIVES

\title{
Neural Binding, CONSCiOUSNeSS, AND MENTAL DISORDERS: COMPLEXITY AS A COMMON ELEMENT
}

\author{
Leonardo P. G. De Assis* \\ Suppes Brain Lab, Center for the Study of Language and Information, \\ Stanford University, Palo Alto, CA, USA
}

\begin{abstract}
Concepts from complex systems have been widely used in model building and experiments in neuroscience. This article presents a short overview of synchronization and chaos theory in studies of perception, and consciousness. In addition, these findings seem to have serious implications for the understanding of mental disorders. Finally some consequences for the future of psychiatry are discussed.
\end{abstract}

Key words: Synchronization; Dynamical systems; Chaos; Complexity; Psychopathology; Mental disorders

\section{INTRODUCTION}

The human brain has a remarkable ability to manage the body functions, and process the information that allows us to act on a changeable external environment. To achieve these functions, it developed a large computing power during evolution as well as specialized functions, such as cognition, emotion, and consciousness. With approximately 86 billion neurons (Herculano-Houzel, 2009), each neuron having approximately hundreds up to thousands of synapses, the brain has high structural complexity, and non-linearity, that endows it with a rich dynamic.

Additionally, the brain is a physical system composed of neurons that communicate with each other through electrical discharges. With that in mind, it is no surprise that the main model used in neuroscience to describe the behavior of neurons was inspired by an electrical circuit model (Hodgkin \& Huxley, 1952; 1990). The brain can also be seen as a physical system, since to its functions are dynamical results of its components, the neurons (McKenna et al., 1994; Rolls \& Traves, 1990; Hertz, Krogh \& Palmer, 1991). For this reason neuroscience has been using concepts and models originating from physics as a fundamental part of the way to understand the brain.

In this short overview I will focus on two concepts coming from areas of mathematical physics, complexity theory or complex systems (Érdi, 2007; Mainzer, 2007; Mitchell, 2009; Holland, 2014), which aim to understand systems with the characteristics described above. According to Singer (2009) the traditional view of how the brain works as compartmentalized, hierarchical, and serial processing organ is illusory. In fact, brain is a distributed system with a

${ }^{*}$ Correspondence to: Leonardo De Assis, e-mail: lpgassis@stanford.edu

Received August 24, 2015; accepted September 10, 2015; Act Nerv Super 57(3-4), 110-121; ISSN-1802-9698 
large number of components that process information in parallel. This change of perspective demands the use of new concepts and tools. In this article, I will show that brain science has already achieved some benefits from using the complexity approach. Concepts such as attractors, chaos, synchronization, self-organization, emergence, complexity measures, resonance, basin of attraction, and others can easily be found in neurosciences literature nowadays (Chialvo, 2010). Another benefit of using complexity theory in neuroscience is is an interdisciplinary research. In this context, complexity theory can act as a bridge between the various disciplines, since it has been used in the study of phenomena not only in physics, but also in chemistry, in biology, linguistics, economics, psychology and others. In particular, I will address the use of concepts related to synchronization and chaos theory in the studies of perception, consciousness, and mental disorders.

\section{SYNCHRONIZATION AND BINDING PROBLEM}

The so-called "Neural Binding Problem" is an important and unresolved problem in neuroscience (Burwick, 2014). This problem stems from the fact that neuroscientists claim that different brain locations represent the distinct features that are present in sensory stimuli. For instance, after we look a red triangle, one neuron assembly processes the red color, and another assembly processes the triangular shape.

From this perspective there is a question: how the brain binds these distinct neural representations and allows the notion of one single object. This problem becomes more apparent when we present to the brain more than one object, for example, a red triangle, and a yellow square (Raffone \& Van Leeuwen, 2001b). How does the brain keep track of the features triangle, square, red, and yellow, in a way that avoid an incorrect combination?

If we consider a computer system such as a PC this would be no problem, due to the fact that the system allocates each piece of information in a specific memory address. However, in a distributed system, such as the brain, the solution is not trivial. Moreover, it is not a matter of finding a mechanism that works across a generic distributed system, but one that is appropriate for the brain. It is relevant to note, that when I say that every sensory feature would be identified with a specific assembly of neurons, it does not imply that these neurons need to be located next to each other.

An intuitive solution would be to imagine the existence of dedicated assembly connecting the different features represented in distinct brain locations. Another alternative would be the presence of specialized synapses connecting distinct feature assemblies. However, these options were discarded, since these solutions would involve creating specialized circuits in the brain. As a consequence of this inflexibility, the demand for memory storage and processing would be greater than even the brain's capacity.

The hypothesis to solve the binding problem that has received the most attention involves the synchronization of neuronal assemblies, as the binding mechanism (Von Der Malsburg, 1995; Singer, 1999; Uhlhaas et al., 2009).

Notably, a rich dynamics such as, resonance, synchronization, solitons, chaos, among others arises from the nonlinear nature of these systems (Arenas et al., 2008; Chen, 2008a; Wang, 2002). Another way to describe this fact is to say that, these phenomena depend on the way in which the components of those systems are interconnected. In a complex network, such as the brain in addition to its large number of components, there are varieties of topologies that give rise to different complex dynamic patterns (Boccaletti et al., 2006; Arenas et al., 2008). For the solution of binding problem, synchronization makes possible the emergence of new "virtual neural assemblies" in the brain, without creating permanent neural circuits.

This suggestion received the support of various experiments carried out in animals, and humans. These experiments showed the presence of higher correlation between oscillations of neuron assemblies that represent the features of the object that gave rise to the stimulus. These 
studies also showed that depending on the oscillation frequency, synchronization has characteristic spatial range, specific brain areas, and links to different cognitive functions (Engel, Fries, \& Singer, 2001; Fries, Neuenschwander et al., 2001; Hipp, Engel, \& Siegel, 2011; de Oliveira, Thiele, \& Hoffmann, 1997; Varela et al., 2001).

According to this classification, alpha waves $(8-12 \mathrm{~Hz})$ were associated with all cortical regions, mainly thalamus and hippocampus, with a long-range synchronization. Synchronization in these frequencies is associated with attentional processes. Beta waves (13$30 \mathrm{~Hz}$ ) were associated with all cortical regions, sub thalamic nucleus, basal ganglia, hippocampus, olfactory bulb, and they also have long-range synchronization. The principal associated cognitive processes are perception, attention, motor control, sensory gating, topdown control, and consciousness. Finally, Gamma waves $(30-200 \mathrm{~Hz})$ with their long-range synchrony act in all cortical regions: the hippocampus, retina, tectum, basal ganglia, and olfactory bulb (Uhlhaas, \& Singer, 2013). Among the brain functions associated with this frequency are perception, attention, memory, consciousness, synaptic plasticity, and motor control.

Although several studies have shown that this proposal has a number of advantages, today no one knows for sure, which specific mechanism the brain uses to accomplish this binding by synchronization. In searching for an appropriate neural synchronization mechanism several theoretical models have been developed (Malsburg, 1995; Jalili, 2009; Suppes et al., 2012; Vassilieva et al., 2011; Zavaglia et al., 2012; Burwick, 2014; Raffone \& Van Leeuwen, 2001b). These models have shown that employing synchronization it is possible to perform various types of computing. Examples of these types of computing are recognition of images (vision), recognition of attributes in sentences (language), logical operations, attention, associative memory, as well as perform all those functions under similar noise conditions of those found in the brain.

\section{NOT BINDING - GLOBAL CHAOS?}

Another view of how to deal with the binding problem suggests using the theory of deterministic chaos. Chaotic systems are characterized by stochastic behavior. However, they can be described by deterministic equations (Scott, 2007; Tabor, 1989). This means that in principle, it may be possible to determine the system evolution during the time. In addition, chaotic systems have the property of being sensitive to initial conditions, which implies that very slight changes in initial conditions can result in very different solutions. According to this proposal, the binding problem could be a pseudo-problem. The information related to an external object would be globally represented in the brain by a special dynamic neural pattern associated with that stimulus. (Raffone, \& van Leeuwen, 2001a; Tsuda, 2001; Azhar et al., 2005; Freeman, \& Barrie, 2001; Freeman, Gaál., \& Jorsten, 2003).

In the same way as in the approaches of the previous section, these hypotheses presented use of synchronization as a fundamental component. But it seems that in these models the main mechanisms responsible for the integration of different features are the low frequency oscillations, which propagate over long distances in the brain. The different stimuli are combined in these low-frequency oscillations in the form of resonances (Freeman \& Barrie, 2001; Freeman, Gaál, \& Jorsten, 2003). In a similar way, in Raffone \& Van Leeuwen (2001b), article the authors made an evaluation of the limitations of traditional proposals, based on periodic oscillations, and they suggested a model for the binding based on chaotic solutions. In addition, according to these authors, the because of increased sensitivity chaos increases the representation capability. 


\section{RELATION WITH CONSCIOUSNESS}

There is a large literature focused on the relationship between binding and consciousness, since the binding problem addresses the integration of different sensory features, because consciousness requires the integration of various sensory experiences (Bob, 2011; 2015; Cleeremans, 2003; Crick, \& Koch, 1990; Feldman, 2013; Revonsuo, 1999; Singer, 2001; Treisman, 1996; Zmigrod \& Hommel, 2011). In these works, synchronization is the basis for binding mechanism.

However, the existence of synchronization is not a sufficient condition for identification of consciousness in brain signals. For instance, it is known that neural synchronization is possible in situations where people are not conscious. This conclusion is hardly surprising, since even in situations where people are not conscious the existence of feature binding is expected. Currently there is an intense debate about the possibility that some animals may have consciousness (Baars, 2005; Boly et al., 2013; Droege \& Braithwaite, 2015; Fabbro et al., 2015; Seth et al., 2005). Although this discussion is still in progress, there is no doubt that many animals are capable of forming one integral image of objects observed in the environment. In other words animals have ability of binding different features in their brains. The current opinion is that consciousness is part of an advanced step in the evolution process, and therefore is a kind of higher manifestation of binding. In this context, although the binding (synchronization) is not a sufficient condition for the existence of consciousness, it is considered a necessary one.

For instance, one idea to measure consciousness that is currently under consideration uses the concept of mathematical information as a measure of consciousness (Tononi, 2004; 2005; 2007; 2008; Tononi \& Edelman, 1998; Tononi \& Sporns, 2003; Tononi, Edelman \& Sporns, 1998). According to this proposal, the brain is a complex system that exhibits different degrees of organization depending on the function being performed at every moment (Tononi, Edelman \& Sporns, 1998). As a result, this hypothesis assumes that during a state of consciousness, the brain has the highest levels of information. In this case, the information generated by the brain as a whole is greater than the sum of the information generated by each subsystem.

However, various authors have criticized the implementation of this proposal. According to them there are mathematical inconsistencies (Casali et al., 2013; Dehaene et al., 2014; Seth, Barrett \& Barnett 2011; Seth et al., 2006). Most likely, the establishment of a measure of consciousness represents a significant step towards the establishment of a science of consciousness. However, the measure of consciousness is an index, and as an index it is unable to explain the mechanism that generates consciousness. For this purpose, the use of chaos concepts, and synchronization may become inevitable.

\section{SYNCHRONIZATION AND MENTAL DISORDER}

It seems that significant impairments in our perception or consciousness systems may implicate a mental disorder. In recent years, several studies have been reported where monitoring of neural synchronization activity was used as a tool to identify differences in the neural patterns between healthy subjects and others with mental disorders. For example, these studies have shown that schizophrenia arises due to the defective functional connections in networks generated through synchronization. (Uhlhaas, Roux, \& Singer, 2013; Saalmann et al., 2012; Ronenwett \& Csernansky, 2010; Yu et al., 2014)

In particular, these studies have shown that synchronization in the beta frequency band in thalamocortical region was correlated with working memory. In addition, it was found that an activity reduction of mediodorsal thalamus and thalamus cortical areas leads to a deficiency in the processes of acquisition and maintenance of information. (Uhlhaas, Roux, \& Singer, 2013). Besides the beta frequency synchronization, these studies showed correlation in the gamma 
band with schizophrenia (Spencer et al., 2004; 2008; 2009; Uhlhaas et al. 2009; 2010; Lee et al., 2003; Wang et al., 2015).

In addition, various studies have reported that changes in synchronization patterns may be associated also with other disorders. Some of these studies have investigated such correlations in specific disorders such as, ADHD (Groom et al., 2010), bipolar disorder (Atagun et al., 2011; Chen et al., 2008b; Tan et al., 2014; O'Donnell et al., 2004; Özerdem et al., 2010; Özerdem et al., 2011), Alzheimer (Dauwels et al., 2010), first-episode psychosis (Flynn et al., 2008), mild cognitive impairment (Missonnier et al., 2006), Parkinson's disease (Hammond, Bergman, \& Brown, 2007) or traumatic brain injury (Leon-Carrion et al., 2012). Other studies have shown the existence of this association between deficiencies in synchronization and various types of disorders, for example in Alzheimer's disease, mild cognitive impairment, attention, bipolar disorder and obsessive-compulsive disorder (Basar \& Guntekin, 2013; Koenig et al., 2005; Leocani et al., 2001; Uhlhaas \& Singer, 2006).

\section{DIAGNOSIS AND TREATMENT}

Until recently, unlike other medical areas, laboratory tests were not used in psychiatry as a method of diagnosis. Basically interviews and questionnaires were the unique ways to identify a mental disorder. This scenario is changing, and knowledge about the synchronization patterns may playe an important role in this change.

For example, Leuchter et al. (2015) investigated the relationship of synchronization and depressive disorders, and suggested the use of neuromodulation as part of the therapy. In another study Jamal et al. (2014) studied synchrony for the classification of autism spectrum. Also other studies investigated the use of synchrony as a tool for diagnosis purposes (Strelets et al., 2006; Singer, 2012; Peled, 2004; 2012a; 2012b; Gandal et al., 2012; Hinkley et al., 2011; Adeli \& Lichtenstein, 2011; Dauwels et al., 2010; Boutros et al., 2008 ).

In addition to the diagnosis, various techniques have been proposed using synchronization measures as a validation assessments for the treatment of mental disorders, for example using transcranial magnetic stimulation (Cohen et al., 1999; D'Alfonso et al., 2002; Hoffman et al., 2003; Jandl et al., 2005; Jin et al., 2006; Fitzgerald et al., 2008; Schneider et al., 2008; Freitas et al., 2009; Prikryl, 2011; Leuchter et al., 2013; Baruth et al., 2010), electroconvulsive therapy (Okazaki et al., 2015), Deep Brain stimulation (Beudel et al, 2015; Fitzgerald, 2011), to check the effectiveness of Deep Brain Stimulation surgery (Quraan et al, 2014), neurofeedback (Pineda, Juavinett \& Datko, 2012), electrical stimulation (Jiménez et al., 2013), to check the effectiveness of a drug treatment via EEG analysis (Kikuchi et al., 2007) or functional neuroanatomy of neural oscillations (Ford et al., 2007).

\section{CHAOS AND MENTAL DISORDERS}

In 1992 Freeman has suggested the use of complexity theory for the study of schizophrenia and mood instability in bipolar disorders. (Freeman, 1992). According to Freeman, chaos underlies the brain's ability to respond flexibly to the outside world and to generate a rich variety of patterns, including those related to creativity. Therefore according ot his findings the unpredictable underlying order in chaotic systems allows to model the complex behavior observed in the brain.

In fact, the dynamics of the underlying attractor reconstructed from the EEG data seems to be chaotic in nature. Freeman's later findings led to the hypothesis that each area of the brain, instead of each stimulus, has a chaotic attractor. A specific sensory stimulus leads the system to a localized region within the attractor associated with a particular stimulus. Furthermore, the attractors themselves must change as a result of new experiences. 
Freeman also suggested a possible association of presence of chaos in the brain and the observed unpredictability in the mental and behavioral events (Namikawa, Nishimoto, \& Tani, 2011). Those observations were correlated with changes in the spatiotemporal patterns of activity of chaotic systems (Freeman, 1999).

Other studies suggest a relationship of chaotic dynamics with a wide range of cognitive phenomena connected with characteristic changes during the manifestations of mental disorders such as depression or schizophrenia (Gottschalk et al., 1995; Barton, 1994; Huber et al., 1999; Korn \& Faure, 2003; Melancon et al., 2000; Paulus \& Braff, 2003; Freeman, 2011; Bob et al., 2009). There are also works that attribute the chaotic transitions to dissociative states in psychopathological conditions (Bob, 2009; Bob \& Louchakova, 2015). They propose hypotheses relating the process of dissociation with the chaotic dynamics generated by discrete maps (Putnam, 1997) suggesting that the chaotic transitions may be related to dissociative states in the brain (Bob, 2003; 2007). In addition, there are studies linking bipolar disorder with chaos (Gottschalk, Bauer \& Whybrow, 1995; Bahrami et al., 2005; Buckjohn et al., 2010; Hadaeghi et al., 2013). All these observations suggest a special role for the chaotic dynamics in various mental processes.

\section{CONCLUSION}

The basic intent of this overview has been to show that complexity may represent an essential part of future research in neuroscience, and may have special applications for the understanding of mental disorders. Synchronization appears as a unifying element in studies of "binding problem" mainly related to perceptual processes but also in principle related to basic mechanisms of consciousness and mental disorders. In this scenario mental disorders may be interpreted in terms of abnormal synchronization patterns among different brain regions. From this observation, a new understanding of the physical-chemical mechanisms in the brain responsible for these anomalies may emerge.

In the same way, the knowledge gained regarding the brain's chaotic properties has opened new directions, mainly linked to understanding of the systemic nature of brain functions and new insights into the brain stochastic patterns and chaotic dynamics in the brain that may be specifically associated with mental disorders.

Another important consequence is the use of mathematical models that may enable to describe various experimental data and help in clinical practice regarding predictions and treatment strategies.

\section{ACKNOWLEDGEMENTS}

This research was supported by Patrick Suppes Gift Funds. I would like to express my indebtedness to Prof. Suppes (1922-2014), who suggested me this research topic. I would also like to show my gratitude to Prof. John Perry, and Prof. De Barros for providing their insights and expertise that greatly have been helping my research.

\section{REFERENCES}

Arenas, A., Díaz-Guilera, A., Kurths, J., Moreno, Y., \& Zhou, C. (2008). Synchronization in complex networks. Physics Reports, 469(3), 93-153.

Atagun, I., Ozerdem, A., Guntekin, B. and Bas,ar, E. (2011). Evoked and event related theta oscillations are decreased in drug-free euthymic bipolar patients. In: Society of Biological Psychiatry 66th Annual Meeting, 12-14 May 2011, Hyatt Regency, San Francisco, CA, p. 975. 
Adeli, H., \& Lichtenstein, A. G. (2011). Automated EEG-based diagnosis of the neurological and psychiatric disorders. In Systems, Signals and Image Processing (IWSSIP), 2011 18th International Conference on (pp. 1-4). IEEE.

Azhar, H., Iftekharuddin, K., \& Kozma, R. (2005). A chaos synchronization-based dynamic vision model for image segmentation. In Neural Networks, 2005. IJCNN'05. Proceedings. 2005 IEEE International Joint Conference on (Vol. 5, pp. 3075-3080). IEEE.

Baars, B. J. (2005). Subjective experience is probably not limited to humans: The evidence from neurobiology and behavior. Consciousness and Cognition, 14(1), 7-21.

Bahrami, B., Seyedsadjadi, R., Babadi, B., \& Noroozian, M. (2005). Brain complexity increases in mania. Neuroreport, 16(2), 187-191.

Barton, S. (1994). Chaos, self-organization, and psychology. American Psychologist, 49(1), 5.

Basar, E., \& Guntekin, B. (2013). Review of delta, theta, alpha, beta, and gamma response oscillations in neuropsychiatric disorders. Clinical Neurophysiology, 62, 303-341.

Bob, P. (2003). Dissociation and neuroscience: history and new perspectives. International Journal of Neuroscience, 113(7), 903-914.

Bob, P. (2007). Chaotic patterns of electrodermal activity during dissociated state released by hypnotic abreaction. International Journal of Clinical and Experimental Hypnosis, 55, 435-436.

Bob, P. (2009). Chaos, cognition and disordered brain. Activitas Nervosa Superior, 50(4).

Bob, P., Susta, M., Chladek, J., Glaslova, K., \& Palus, M. (2009). Chaos in schizophrenia associations, reality or metaphor? International Journal of Psychophysiology, 73(3), 179-185.

Bob, P. (2011). Brain, mind and consciousness: advances in neuroscience research. Springer Science \& Business Media, New York.

Bob, P. (2013). Dissociation, epileptiform discharges and chaos in the brain: toward a neuroscientific theory of dissociation. ANS: The Journal for Neurocognitive Research, 54(3-4).

Bob, P. (2014). Dissociation, Chaos and Transcendent Function. Activitas Nervosa Superior, 55(4).

Bob, P. (2015). The Brain and Conscious Unity: Freud's Omega. New York, NY: Springer New York.

Bob, P., \& Louchakova, O. (2015). Dissociative states in dreams and brain chaos: Implications for creative awareness. Frontiers in Psychology, 6, 1353.

Boccaletti, S., Latora, V., Moreno, Y., Chavez, M., \& Hwang, D. U. (2006). Complex networks: Structure and dynamics. Physics reports, 424(4), 175-308.

Boly, M., Seth, A. K., Wilke, M., Ingmundson, P., Baars, B., Laureys, S., et al. (2013). Consciousness in humans and non-human animals: recent advances and future directions. Frontiers in psychology, 4.

Boutros, N.N., Arfken, C., Galderisi, S., Warrick, J., Pratt, G. and Iacono, W. (2008) The status of spectral EEG abnormality as a diagnostic test for schizophrenia. Schizophrenia Research, 99 (1-3): 225-237.

Buckjohn, C. N. D., Siewe, M. S., Tchawoua, C., \& Kofane, T. C. (2010). Synchronization of chaotic and nonchaotic oscillators: Application to bipolar disorder. Physics Letters A, 374(35), 3646-3655.

Burwick, T. (2014). The binding problem. Wiley Interdisciplinary Reviews: Cognitive Science WIREs Cogn Sci, 5, 305-315.

Casali, A. G., Gosseries, O., Rosanova, M., Boly, M., Sarasso, S., Casali, K. R., ... \& Massimini, M. (2013). A theoretically based index of consciousness independent of sensory processing and behavior. Science translational medicine, 5(198), 198ra105-198ra105.

Chen, M. (2008a). Chaos synchronization in complex networks. Circuits and Systems I: Regular Papers, IEEE Transactions on, 55(5), 1335-1346.

Chen, S. S., Tu, P. C., Su, T. P., Hsieh, J. C., Lin, Y. C., \& Chen, L. F. (2008b). Impaired frontal synchronization of spontaneous magnetoencephalographic activity in patients with bipolar disorder. Neuroscience letters, 445(2), 174-178.

Chialvo, D. R. (2010). Emergent complex neural dynamics. Nature physics, 6(10), 744-750.

Cleeremans, A. E. (2003). The unity of consciousness: Binding, integration, and dissociation. Oxford University Press.

Crick, F., \& Koch, C. (1990). Towards a neurobiological theory of consciousness. In Seminars in the Neurosciences (Vol. 2, pp. 263-275). Saunders Scientific Publications. 
Dauwels, J., Vialatte, F., Musha, T. and Cichocki, A. (2010). A comparative study of synchrony measures for the early diagnosis of Alzheimer's disease based on EEG. Neuroimage, 49 (1): 668-693.

Dauwels, J., Vialatte, F., Musha, T. and Cichocki, A. (2010). A comparative study of synchrony measures for the early diagnosis of Alzheimer's disease based on EEG. Neuroimage, 49 (1): 668-693.

Dehaene, S., Charles, L., King, J. R., \& Marti, S. (2014). Toward a computational theory of conscious processing. Current opinion in neurobiology, 25, 76-84.

Droege, P., \& Braithwaite, V. A. (2015). A Framework for Investigating Animal Consciousness. Ethical Issues in Behavioral Neuroscience, 79-98.

Engel, A. K., Fries, P., \& Singer, W. (2001). Dynamic predictions: oscillations and synchrony in top-down processing. Nature Reviews Neuroscience, 2(10), 704-716.

Érdi, P. (2007). Complexity explained. Springer Science \& Business Media.

Fabbro, F., Aglioti, S. M., Bergamasco, M., Clarici, A., \& Panksepp, J. (2015). Evolutionary aspects of selfand world consciousness in vertebrates. Frontiers in human neuroscience, 9.

Feldman, J. (2013). The neural binding problem (s). Cognitive neurodynamics, 7(1), 1-11.

Flynn, G., Alexander, D., Harris, A., Whitford, T., Wong, W., Galletly, C., Silverstein, S., Gordon, E. and Williams, L.M. (2008). Increased absolute magnitude of gamma synchrony in first-episode psychosis. Schizophrenia Research, 105(1-3): 262-271.

Freeman, W. (1992). Chaos in psychiatry. Biological Psychiatry, 1079-1081.

Freeman, W. J. (1999). Consciousness, intentionality and causality. Journal of Consciousness Studies, 6(11-12), 143-172.

Freeman, W. J., \& Barrie, J. M. (2001). Chaotic oscillations and the genesis of meaning in cerebral cortex. In NATO Advanced Study Institute on Nonlinear Dynamics in the Life and Social Sciences, Apr-May, 2000, Lomonosov Moscow State University, Moscow, Russia. IOS Press.

Freeman, W. J. W. (2011). The emergence of mind and emotion in the evolution of neocortex. Rivista di psichiatria, 46(5-6).

Freeman, W. J., Gaál, G., \& Jorsten, R. (2003). A neurobiological theory of meaning in perception Part III: Multiple cortical areas synchronize without loss of local autonomy. International Journal of Bifurcation and Chaos, 13(10), 2845-2856.

Fries, P., Neuenschwander, S., Engel, A. K., Goebel, R., \& Singer, W. (2001). Rapid feature selective neuronal synchronization through correlated latency shifting. Nature neuroscience, 4(2), 194-200.

Gandal, M. J., Edgar, J. C., Klook, K., \& Siegel, S. J. (2012). Gamma synchrony: towards a translational biomarker for the treatment-resistant symptoms of schizophrenia. Neuropharmacology, 62(3), 15041518.

Gottschalk, A., Bauer, M. S., \& Whybrow, P. C. (1995). Evidence of chaotic mood variation in bipolar disorder. Archives of general psychiatry, 52(11), 947-959.

Groom, M.J., Cahill, J.D., Bates, A.T., Jackson, G.M., Calton, T.G., Liddle, P.F. and Hollis, C. (2010). Electrophysiological indices of abnormal error-processing in adolescents with attention deficit hyperactivity disorder (ADHD). Journal of Child Psychology and Psychiatry, 51(1): 66-76.

Hadaeghi, F., Golpayegani, M. R. H., \& Moradi, K. (2013). Does “crisis-induced intermittency" explain bipolar disorder dynamics?. Frontiers in computational neuroscience, 7.

Hammond, C., Bergman, H., \& Brown, P. (2007). Pathological synchronization in Parkinson's disease: networks, models and treatments. Trends in neurosciences, 30(7), 357-364.

Herculano-Houzel, S. (2009). The human brain in numbers: a linearly scaled-up primate brain. Frontiers in human neuroscience, 3.

Hertz, J., Krogh, A., \& Palmer, R. G. (1991). Introduction to the theory of neural computation (Vol. 1). Basic Books.

Hinkley, L. B., Vinogradov, S., Guggisberg, A. G., Fisher, M., Findlay, A. M., \& Nagarajan, S. S. (2011). Clinical symptoms and alpha band resting-state functional connectivity imaging in patients with schizophrenia: implications for novel approaches to treatment. Biological psychiatry, 70(12), 1134-1142.

Hipp, J. F., Engel, A. K., \& Siegel, M. (2011). Oscillatory synchronization in large-scale cortical networks predicts perception. Neuron, 69(2), 387-396. 
Hodgkin, A. L., \& Huxley, A. F. (1952). A quantitative description of membrane current and its application to conduction and excitation in nerve. The Journal of physiology, 117(4), 500-544.

Hodgkin, A. L., \& Huxley, A. F. (1990). A quantitative description of membrane current and its application to conduction and excitation in nerve. Bulletin of mathematical biology, 52(1-2), 25-71.

Holland, J. H. (2014). Complexity: A very short introduction. Oxford University Press.

Huber, M. T., Braun, H. A., \& Krieg, J. C. (1999). Consequences of deterministic and random dynamics for the course of affective disorders. Biological psychiatry, 46(2), 256-262.

Koenig, T., Prichep, L., Dierks, T., Hubl, D., Wahlund, L. O., John, E. R., \& Jelic, V. (2005). Decreased EEG synchronization in Alzheimer's disease and mild cognitive impairment. Neurobiology of Aging, 26(2), 165-171.

Korn, H., \& Faure, P. (2003). Is there chaos in the brain? II. Experimental evidence and related models. Comptes rendus biologies, 326(9), 787-840.

Jamal, W., Das, S., Oprescu, I. A., Maharatna, K., Apicella, F., \& Sicca, F. (2014). Classification of autism spectrum disorder using supervised learning of brain connectivity measures extracted from synchrostates. Journal of neural engineering, 11(4), 046019.

Jalili, M. (2009). Synchronizing Hindmarsh-Rose neurons over Newman-Watts networks. Chaos: An Interdisciplinary Journal of Nonlinear Science, 19(3), 033103.

Lee, K., Williams, L., Haig, A., \& Gordon, E. (2003). "Gamma (40 Hz) phase synchronicity" and symptom dimensions in schizophrenia. Cognitive Neuropsychiatry, 57-71.

Leocani, L., Locatelli, M., Bellodi, L., Fornara, C., Hénin, M., Magnani, G., et al. (2001). Abnormal pattern of cortical activation associated with voluntary movement in obsessive-compulsive disorder: an EEG study. American Journal of Psychiatry, 158(1), 140-142.

Leon-Carrion, J., Leon-Dominguez, U., Pollonini, L., Wu, M. H., Frye, R. E., Dominguez-Morales, M. R., \& Zouridakis, G. (2012). Synchronization between the anterior and posterior cortex determines consciousness level in patients with traumatic brain injury (TBI). Brain Research, 1476, 22-30.

Leuchter, A. F., Hunter, A. M., Krantz, D. E., \& Cook, I. A. (2015). Rhythms and blues: modulation of oscillatory synchrony and the mechanism of action of antidepressant treatments. Annals of the New York Academy of Sciences, 1344(1), 78-91.

Mainzer, K. (2007). Thinking in complexity: The computational dynamics of matter, mind, and mankind. Springer Science \& Business Media.

Malsburg, C. (1995). Binding in models of perception and brain function. Current Opinion in Neurobiology, $5(4), 520-526$.

Mccarley, R. (2004). Neural synchrony indexes disordered perception and cognition in schizophrenia. Proceedings of the National Academy of Sciences, 17288-17293.

McKenna, T. M., McMullen, T. A., \& Shlesinger, M. F. (1994). The brain as a dynamic physical system. Neuroscience, 60(3), 587-605.

Melancon, G., Joanette, Y., \& Bélair, J. (2000). Chaos, brain, and cognition: Toward a nonlinear order?. Brain and cognition, 42(1), 33-36.

Missonnier, P., Gold, G., Herrmann, F.R., Fazio-Costa, L., Michel, J.P., Deiber, M.P., Michon, A. and Giannakopoulos, P. (2006). Decreased theta event-related synchronization during working memory activation is associated with progressive mild cognitive impairment. Dement. Geriatr. Cogn. Disord., 22(3): 250-259.

Mitchell, M. (2009). Complexity: A guided tour. Oxford University Press.

Namikawa, J., Nishimoto, R., \& Tani, J. (2011). A neurodynamic account of spontaneous behaviour. PLoS Computational Biology, 7(10), e1002221.

O'Donnell, B.F., Hetrick, W.P., Vohs, J.L., Krishnan, G.P., Carroll, C.A. \& Shekhar, A. (2004). Neural synchronization deficits to auditory stimulation in bipolar disorder. NeuroReport, 15, 1369-1372.

de Oliveira, S. C., Thiele, A., \& Hoffmann, K. P. (1997). Synchronization of neuronal activity during stimulus expectation in a direction discrimination task. The Journal of Neuroscience, 17(23), 9248-9260.

Ozdemir, O., Ozdemir, P. G., \& Yilmaz, E. (2014). The butterfly effect in psychiatry: a case example. Journal of Mood Disorders, 4(1), 34-7. 
Özerdem, A., Güntekin, B., Saatçi, E., Tunca, Z., \& Başar, E. (2010). Disturbance in long distance gamma coherence in bipolar disorder. Progress in Neuro-Psychopharmacology and Biological Psychiatry, 34(6), 861-865.

Özerdem, A., Güntekin, B., Atagün, İ., Turp, B., \& Başar, E. (2011). Reduced long distance gamma (28$48 \mathrm{~Hz}$ ) coherence in euthymic patients with bipolar disorder. Journal of affective disorders, 132(3), 325332.

Paulus, M. P., \& Braff, D. L. (2003). Chaos and schizophrenia: does the method fit the madness? Biological Psychiatry, 53(1), 3-11.

Putnam, F. W. (1997). Dissociation in children and adolescents: A developmental perspective. Guilford Press.

Peled, A. (2004). From plasticity to complexity: a new diagnostic method for psychiatry. Medical hypotheses, 63(1), 110-114.

Peled, A. (2012a). Neuroanalysis: A method for brain-related neuroscientific diagnosis of mental disorders. Medical hypotheses, 78(5), 636-640.

Peled, A. (2012b). Personality disorders disturbances of the physical brain. Medical hypotheses, 79(4), 487492.

Raffone, A., \& van Leeuwen, C. (2001a). Chaos and neural coding: is the binding problem a pseudoproblem?. Behavioral and Brain Sciences, 24(05), 826-827.

Raffone, A., \& Van Leeuwen, C. (2001b). Activation and coherence in memory processes: Revisiting the parallel distributed processing approach to retrieval. Connection Science, 13(4), 349-382.

Revonsuo, A. (1999). Binding and the phenomenal unity of consciousness. Consciousness and cognition, 8(2), 173-185.

Rolls, E., \& Traves, A. (1990). Neuronal networks in the brain. Physics World, 3, 31-35.

Ronenwett, W.J., \& Csernansky, J. (2010). Curr. Top Behav. Neurosci. 4, 509-528.

Saalmann, Y.B., Pinsk, M.A., Wang, L., Li, X., \& Kastner, S. (2012). Science 337, 753-756.

Scott, A. C. (2007). The nonlinear universe: chaos, emergence, life. Springer Science \& Business Media.

Seth, A. K., Baars, B. J., \& Edelman, D. B. (2005). Criteria for consciousness in humans and other mammals. Consciousness and cognition, 14(1), 119-139.

Seth, A. K., Barrett, A. B., \& Barnett, L. (2011). Causal density and integrated information as measures of conscious level. Philosophical Transactions of the Royal Society of London A: Mathematical, Physical and Engineering Sciences, 369(1952), 3748-3767.]

Seth, A. K., Izhikevich, E., Reeke, G. N., \& Edelman, G. M. (2006). Theories and measures of consciousness: an extended framework. Proceedings of the National Academy of Sciences, 103(28), 1079910804.

Singer, W. (1999). Neuronal Synchrony: A Versatile Code for the Definition of Relations? Neuron, 24, 496.

Singer, W. (2001). Consciousness and the binding problem. Annals of the New York Academy of Sciences, 929(1), 123-146.

Singer, W. (2009). The brain, a complex self-organizing system. European Review, 17(02), 321-329.

Singer, W. (2012). What binds it all together? Synchronized oscillatory activity in normal and pathological cognition (pp. 57-70). Springer Vienna.

Spencer, K., Salisbury, D., Shenton, M., \& Mccarley, R. (2008). $\gamma$-Band Auditory Steady-State Responses Are Impaired in First Episode Psychosis. Biological Psychiatry, 369-375.

Spencer, K., Niznikiewicz, M., Nestor, P., Shenton, M., \& Mccarley, R. (2009). Left auditory cortex gamma synchronization and auditory hallucination symptoms in schizophrenia. BMC Neuroscience BMC Neurosci, 85-85.

Strelets, V. B., Garakh, Z. V., Novototskii-Vlasov, V. Y., \& Magomedov, R. A. (2006). Relationship between EEG power and rhythm synchronization in health and cognitive pathology. Neuroscience and behavioral physiology, 36(6), 655-662.

Suppes, P., de Barros, J. A., \& Oas, G. (2012). Phase-oscillator computations as neural models of stimulus-response conditioning and response selection. Journal of Mathematical Psychology, 56(2), 95117. 
Tabor, M. (1989). Chaos and integrability in nonlinear dynamics: an introduction. Wiley-Interscience.

Tan, D., Özerdem, A., Güntekin, B., Atagün, M. I., Tülay, E., Karadağ, F., \& Başar, E. (2014). Increased Beta Frequency (15-30 Hz) Oscillatory Responses in Euthymic Bipolar Patients Under Lithium Monotherapy. Clinical EEG and neuroscience, 1550059414561056.

Tillmann, C., Wibral, M., Leweke, M., Kohler, A., Singer, W., Koethe, D., ... \& Uhlhaas, P. (2008). Source localization of high-frequency oscillations reveals widespread reductions in gamma-band activity during perceptual organization in chronic and first-episode schizophrenia. In Soc. Neurosci. Abstr (Vol. 54).

Tononi, G. (2004). An information integration theory of consciousness. BMC neuroscience, 5(1), 42.

Tononi, G. (2005). Consciousness, information integration, and the brain. Progress in brain research, 150, 109-126.

Tononi, G. (2007). The information integration theory of consciousness (pp. 287-299). Blackwell: Malden, MA, USA.

Tononi, G. (2008). Consciousness as integrated information: a provisional manifesto. The Biological Bulletin, 215(3), 216-242.

Tononi, G., \& Edelman, G. M. (1998). Consciousness and complexity. Science, 282(5395), 1846-1851.

Tononi, G., Edelman, G. M., \& Sporns, O. (1998). Complexity and coherency: integrating information in the brain. Trends in cognitive sciences, 2(12), 474-484.

Tononi, G., \& Sporns, O. (2003). Measuring information integration. BMC neuroscience, 4(1), 31.

Treisman, A. (1996). The binding problem. Current opinion in neurobiology, 6(2), 171-178.

Tsuda, I. (2001). Toward an interpretation of dynamic neural activity in terms of chaotic dynamical systems. Behavioral and Brain Sciences, 24(05), 793-810.

Uhlhaas, P., Linden, D., Singer, W., Haenschel, C., Lindner, M., Maurer, K., \& Rodriguez, E. (2006). Dysfunctional Long-Range Coordination of Neural Activity during Gestalt Perception in Schizophrenia. Journal of Neuroscience, 8168-8175.

Uhlhaas, P. J., \& Singer, W. (2006). Neural synchrony in brain disorders: relevance for cognitive dysfunctions and pathophysiology. Neuron, 52(1), 155-168.

Uhlhaas, P., Pipa, G., Lima, B., Melloni, L., Neuenschwander, S., Nikolić, D., \& Singer, W. (2009). Neural synchrony in cortical networks: history, concept and current status. Frontiers in integrative neuroscience, 3, 17.

Uhlhaas, P., \& Singer, W. (2010). Abnormal neural oscillations and synchrony in schizophrenia. Nature Reviews Neuroscience, 100-113.

Uhlhaas, P. J., Roux, F., and Singer, W. (2013) Thalamocortical synchronization and cognition: implications for schizophrenia? Neuron, 77, (6). pp. 997-999.

Uhlhaas, P. J., \& Singer, W. (2013). High-frequency oscillations and the neurobiology of schizophrenia. Dialogues in clinical neuroscience, 15(3), 301.

Varela, F., Lachaux, J. P., Rodriguez, E., \& Martinerie, J. (2001). The brainweb: phase synchronization and large-scale integration. Nature reviews neuroscience, 2(4), 229-239.

Vassilieva, E., Pinto, G., De Barros, J. A., \& Suppes, P. (2011). Learning pattern recognition through quasi-synchronization of phase oscillators. Neural Networks, IEEE Transactions, 22(1), 84-95.

Von Der Malsburg, C. (1995). Binding in models of perception and brain function. Current opinion in neurobiology, 5(4), 520-526.

Yang, A. C., \& Tsai, S. J. (2013). Is mental illness complex? From behavior to brain. Progress in NeuroPsychopharmacology and Biological Psychiatry, 45, 253-257.

Yu, R., Chien, Y. L., Wang, H. L. S., Liu, C. M., Liu, C. C., Hwang, T. J., ... \& Tseng, W. Y. I. (2014). Frequency-specific alternations in the amplitude of low-frequency fluctuations in schizophrenia. Human brain mapping, 35(2), 627-637.

Wang, X. F. (2002). Complex networks: topology, dynamics and synchronization. International Journal of Bifurcation and Chaos, 12(05), 885-916. 
Wang, G., Kydd, R., \& Russell, B. (2015). Quantitative EEG and Low-Resolution Electromagnetic Tomography (LORETA) Imaging of Patients Undergoing Methadone Treatment for Opiate Addiction. Clinical EEG and Neuroscience.

Zavaglia, M., Canolty, R. T., Schofield, T. M., Leff, A. P., Ursino, M., Knight, R. T., \& Penny, W. D. (2012). A dynamical pattern recognition model of gamma activity in auditory cortex. Neural Networks, 28, 114.

Zmigrod, S., \& Hommel, B. (2011). The relationship between feature binding and consciousness: Evidence from asynchronous multi-modal stimuli. Consciousness and cognition, 20(3), 586-593. 